\title{
Composite Hydrogels with the Simultaneous Release of VEGF and MCP-1 for Enhancing Angiogenesis for Bone Tissue Engineering Applications
}

\author{
Lei Nie ${ }^{1,2, *,+}$, Pengbo Chang ${ }^{3,+}$, Meng Sun ${ }^{1,2}$, Haojie Huo ${ }^{2,4}$, Chunxia Zhang ${ }^{2,4}$, \\ Chingching Ji ${ }^{5}$, Xiaoyan Wei ${ }^{6}$, Qiuju Zhou ${ }^{7}$, Peiyin Guo ${ }^{7}$ and Hongyu Yuan ${ }^{1,2}$ \\ 1 College of Life Sciences, Xinyang Normal University, Xinyang 464000, China; \\ sunmeng2010abc@163.com (M.S.); yhongyu92@163.com (H.Y.) \\ 2 Henan Key Laboratory of Tea Plant Biology, Xinyang Normal University, Xinyang 464000, China; \\ huohaojie@139.com (H.H.); zhangchunxia915@163.com (C.Z.) \\ 3 Zhengzhou Technical College, Zhengzhou 450010, China; cpb317@163.com \\ 4 College of Chemistry and Chemical Engineering, Xinyang Normal University, Xinyang 464000, China \\ 5 Institut für Chemie und Biochemie-Physikalische und Theoretische Chemie, Freie Universität Berlin, \\ Takustr. 3, 14195 Berlin, Germany; chi.ji91@gmail.com \\ 6 Max Planck Institute for Molecular Genetics, Ihnestraße 63-73, 14195 Berlin, Germany; \\ weixiaoyan@zedat.fu-berlin.de \\ 7 Analysis \& Testing Center, Xinyang Normal University, Xinyang 464000, China; \\ zhouqiuju@iccas.as.cn (Q.Z.); guopy1991@163.com (P.G.) \\ * Correspondence: nielei@xynu.edu.cn or nieleifu@yahoo.com; Tel.: +86-136-0062-1068 \\ + These authors contributed equally to this work and should be considered co-first authors.
}

Received: 19 October 2018; Accepted: 23 November 2018; Published: 1 December 2018

\begin{abstract}
Rapid new microvascular network induction was critical for bone regeneration, which required the spatiotemporal delivery of growth factors and transplantation of endothelial cells. In this study, the linear poly(D,L-lactic-co-glycolic acid)-b-methoxy poly(ethylene glycol) (PLGA-mPEG) block copolymer microspheres were prepared for simultaneously delivering vascular endothelial growth factor (VEGF) and monocyte chemotactic protein-1 (MCP-1). Then, vascular endothelial cells (VECs) with growth factor loaded microspheres were composited into a star-shaped PLGA-mPEG block copolymer solution. After this, composite hydrogel (microspheres ratio: $5 \mathrm{wt} \%$ ) was formed by increasing the temperature to $37^{\circ} \mathrm{C}$. The release profiles of VEGF and MCP-1 from composite hydrogels in 30 days were investigated to confirm the different simultaneous delivery systems. The VECs exhibited a good proliferation in the composite hydrogels, which proved that the composite hydrogels had a good cytocompatibility. Furthermore, in vivo animal experiments showed that the vessel density and the mean vessel diameters increased over weeks after the composite hydrogels were implanted into the necrosis site of the rabbit femoral head. The above results suggested that the VECs-laden hydrogel composited with the dual-growth factor simultaneous release system has the potential to enhance angiogenesis in bone tissue engineering.
\end{abstract}

Keywords: composite hydrogel; simultaneous release; growth factor; angiogenesis; bone tissue engineering

\section{Introduction}

There was an urgent need of engineered-organ transplantation for treating bone-related diseases, such as the bone tumor, osteoporosis, and osteonecrosis of the femoral head (ONFH). The temporary three-dimensional (3D) multifunctional scaffold has the potential to induce physiological regeneration, 
including angiogenesis and osteogenesis [1-4]. Thereinto, the ingrowth of new blood vessels was critical for stimulating osteogenesis, and the formed capillary network also served as a template for new bone formation [5]. The lack of microvasculature formation remains a key roadblock to apply engineered composites to clinical applications up to now [6]. Several reports proved that many soluble growth factors could regulate vasculogenesis and angiogenesis, mainly including the vascular endothelial growth factor (VEGF), fibroblast growth factor (FGF), insulin-like growth factor (IGF), hepatocyte growth factor (HGF), platelet-derived growth factor-BB (PDGF-BB), monocyte chemoattractant protein-1 (MCP-1), etc. [7-11]. VEGF was one of the most important growth factors across all stages of neovascularization, mainly through initiating the sprouting of existing blood vessels by mitogenic and chemotactic effects, and facilitating the recruitment of circulating endothelial progenitor cells $[9,10]$. Furthermore, VEGF was also important for maintaining the survival of blood vessels, the data indicated that the withdrawal of VEGF caused vascular collapse and regression [9-11]. As a matter of fact, a sustaining release of VEGF at least for a period of four weeks could improve the maturing and stabilizing of newly formed blood vessels [12]. On the other hand, the VEGF could not only stimulate the proliferation, migration, and survival of VECs, but also enhanced the alkaline phosphatase activity, mineralized tissue production, and adhered molecule, which played an important role in fracture healing $[13,14]$. Because of its short half-life and enzymatic degradation of the growth factors, some side effects were unavoidable by using very high doses. Thus, an effective delivery system was significant.

On the other hand, new bone formation and angiogenesis were intimately connected during tissue regeneration [5]. Many studies confirmed that bone tissue regeneration could be ameliorated via the spatiotemporal release of VEGF and bone growth factors, for instance, bone morphogenetic protein-2 (BMP-2) [15-17]. MCP-1, known as chemokine (C-C motif) ligand 2 (CCL2), was an arteriogenic factor that could induce a remarkable host vascularization response at an early stage, and it was clear that the dual delivery of MCP-1 and VEGF induced the mural cell recruitment and improved survival of endothelial cells (ECs) [4]. In addition, it has been shown that early-stage growth factors could have synergy effects on late-stage factors and vice versa when presented simultaneously [18]. In our previous reports, the biodegradable block copolymer microspheres were proven to be an effective delivery vehicle for encapsulation and the controlled release of growth factors $[19,20]$. The release profile of the growth factor from microspheres could be regulated by adjusting the degradability rate of microspheres. Therefore, a different simultaneous delivery system of dual-growth factors could be facilely constructed through compositing different growth factor loaded microspheres together [21,22]. It was also shown that the injectable hydrogel composited with the delivery system has the potential for treating early-stage osteonecrosis of the femoral head, and such an injection treatment performed many advantages compared to traditional engineered scaffold replacement, including surgical convenience, and lower side effects, etc. [23-27].

In this work, VEGF and MCP-1 were loaded into different linear poly(D,L-lactic-co-glycolic acid)-b-methoxy poly(ethylene glycol) (PLGA-mPEG) block copolymer microspheres with different $[\mathrm{LA}] /[\mathrm{GA}]$ ratios respectively. Then, the growth factors loaded microspheres were composited with star-shaped PLGA-mPEG block copolymer solution, after that, the composite hydrogel was obtained via adjusting the temperature to $37^{\circ} \mathrm{C}$. According to the formulation of microspheres in hydrogels, different delivery systems were designed. At the same time, the vascular endothelial cells (VECs) were also loaded into composite hydrogel during the hydrogel formation process (Figure 1). The release profiles of VEGF and MCP-1 were investigated by ELISA kits, the proliferation of VECs in composite hydrogels was also evaluated. Finally, vascularization followed by new bone formation was investigated via in vivo animal experiments. 
(a)

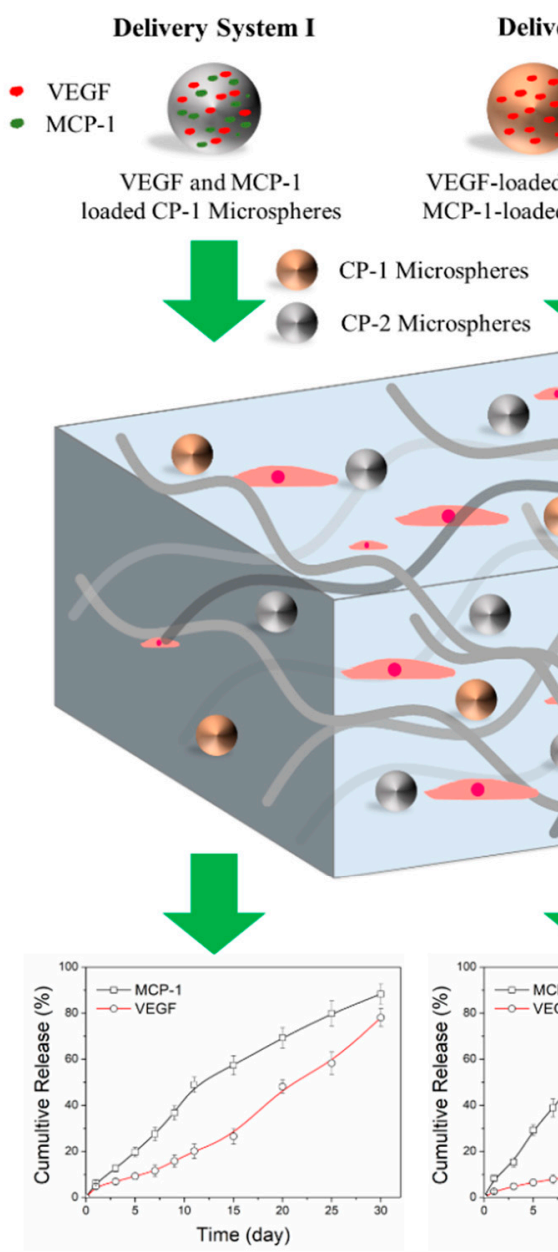

Delivery System II

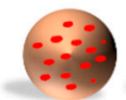

VEGF-loaded CP-1 Microspheres MCP-1-loaded CP-2 Microspheres
Delivery System III

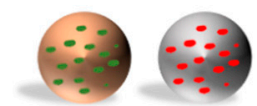

MCP-1-loaded CP-1 Microspheres VEGF-loaded CP-2 Microspheres

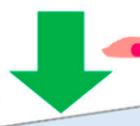

Vascular Endothelial Cells

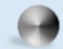
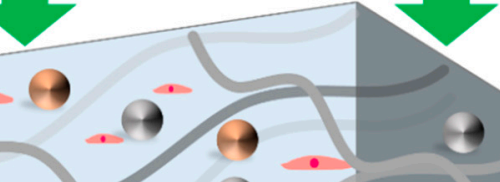

.
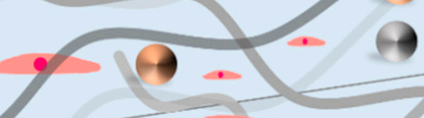

$\rightarrow 0$

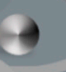

$+$

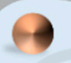

$\theta$

9 $\theta \quad 10$

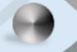

$19 \theta$

-

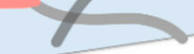

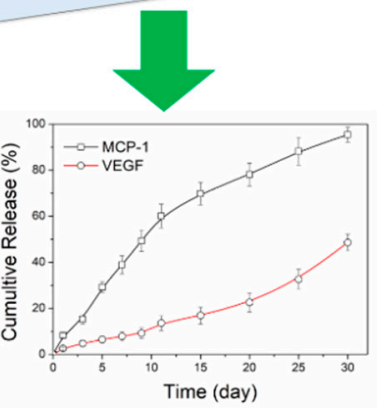

(b)

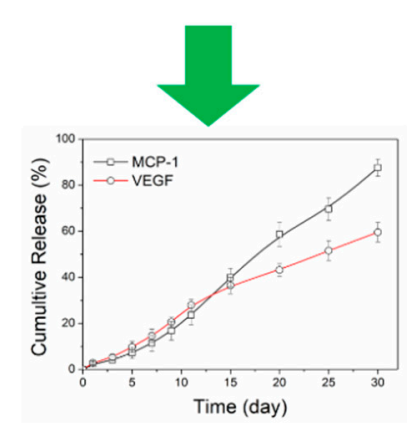

(c)

Figure 1. The schematic illustration for the preparation of composite hydrogel by using the VEGF and MCP-1 simultaneous delivery system (different release profiles of growth factor depend on time) and encapsulating VECs. Different microspheres loaded with VEGF and MCP-1 were composited into a star-shaped block copolymer solution (using simulation body fluid), at the same time, VECs were added into, then cells-laden composite hydrogel was formed at $37^{\circ} \mathrm{C}$. (a) VEGF and MCP-1 release profiles from delivery system I; (b) VEGF and MCP-1 release profiles from delivery system II; (c) MCP-1 and VEGF release profiles from delivery system III.

\section{Materials and Methods}

\subsection{Materials}

D,L-lactide (LA), glycolide (GA), monomethoxy poly(ethylene glycol) (mPEG, $\left.M_{n}=550\right)$, and stannous octoate $\left(\mathrm{Sn}(\mathrm{Oct})_{2}\right)$ were purchased from Sigma Chemical Co. Ltd. (Poole, U.K.). VEGF was obtained from ThermoFisher Scientific Co., Ltd. (Shanghai, China), MCP-1 was obtained from Sino Biological Co., Ltd. (Shanghai, China), and ELISA kits for VEGF and MCP-1 were purchased from Peprotech (Rocky Hill, NJ). All other chemicals were purchased from China National Medicines Corporation Ltd. (analytical grade).

\subsection{Microspheres Preparation}

The linear PLGA-mPEG block copolymers (PLGA $1320-$ mPEG $_{550}$ copolymer with an [LA]/[GA] ratio of 1 , designated as $\mathrm{CP}-1$, and $\mathrm{P}\left(\mathrm{LA}_{3} \mathrm{GA}\right)_{1320}-\mathrm{mPEG}_{550}$ copolymer with an [LA]/[GA] ratio of 3, designated as CP-2) were synthesized (Supplementary Materials) [24]. The VEGF and MCP-1 loaded $\mathrm{CP}-1$ microspheres, VEGF loaded $\mathrm{CP}-1$ microspheres, MCP-1 loaded CP-1 microspheres, 
VEGF loaded CP-2 microspheres, and MCP-1 loaded microspheres were fabricated by using a double-emulsion method according to our previous works (Supplementary Materials) [20]. The morphology of microspheres was examined by Cold Field Scanning Electron Microscopy (SEM, S4800).

\subsection{Composite Hydrogels Preparation}

The star-shaped PLGA-mPEG block copolymer (star-P(LA 3 GA) ${ }_{1320}-\mathrm{mPEG}_{550}$ with an [LA]/[GA] ratio of 3) was synthesized (Supplementary Materials) [24]. The prepared star-shaped block copolymer was dissolved into simulation body fluid ( $40 \mathrm{wt} \%$ ) to obtain a copolymer solution at $10-20{ }^{\circ} \mathrm{C}$, then the prepared growth factors loaded microspheres were added into copolymer solution, next, the composite hydrogels were produced at $37^{\circ} \mathrm{C}$. In this paper, three delivery systems were designed (Figure 1, Table S1). The release profiles of VEGF and MCP-1 were tested by ELISA kits.

\subsection{In Vitro Cytocompatibility}

VECs (obtained from ATCC ${ }^{\circledR}$ CRL-4053 ${ }^{\mathrm{TM}}$ ) were grown in Dulbecco's modified Eagle's medium (DMEM) supplemented with $10 \%$ fetal bovine serum, $100 \mathrm{U} \mathrm{mL}^{-1}$ penicillin, and $100 \mu \mathrm{g} \mathrm{mL}$ streptomycin (all purchased from Gibco, Invitrogen, N.Y., USA) under a humidified atmosphere of $5 \%$ $\mathrm{CO}_{2}$ at $37^{\circ} \mathrm{C}$. During the composite hydrogel preparation, VECs and microspheres were composited to obtain the VECs-laden composite hydrogel. The VECs viability in composite hydrogels at the different days was characterized by using a CCK-8 assay. At the same time, the VECs-laden composite hydrogels were removed from the culture media after 3 days, the phalloidin-FITC $(50 \mu \mathrm{g} / \mathrm{mL}$, Invitrogen, N.Y., USA) and 4,6-diamidino-2-phenylindole (DAPI, $10 \mu \mathrm{g} / \mathrm{mL}$, Invitrogen, NY, USA) were used to stainf the VECs to investigate the proliferation of the VECs; the pink color was used to indicate cytoskeleton, the blue color was used to indicate the nucleus (Supplementary Materials).

\subsection{In Vivo Studies}

\subsubsection{Animal Experiments}

Based on the microstructure, composition, and remodeling of rabbit bones, rabbits showed many advantages for bone regeneration experiments [28,29]. The Japanese rabbits were used in this study. For all studies, rabbits were anesthetized with $2 \%$ pentobarbital sodium $(30 \mathrm{mg} / \mathrm{kg})$ via the rabbit ear marginal vein, and the animals were kept in the Institute of Experimental Animals of Wuhan Hanyang Hospital, in accordance with the institutional guidelines for care and use of laboratory animals. The animal experiments were approved by the Animal Ethical Committee of Wuhan Hanyang Hospital (Permit number 00027467). The femoral head necrosis model in the right-posterior position of rabbits was constructed firstly, then the star-shaped block copolymer solution composited with VECs and growth factor loaded microspheres were injected into necrosis site, the composite hydrogel was formed inside in $5 \mathrm{~min}$. The rabbits were treated at week 4 and 8 for angiogenesis evaluations (Supplementary Materials).

\subsubsection{X-Ray Scanning}

After the surgery, $\mathrm{X}$-ray scanning of the rabbit femoral head in the right-posterior position was performed using an X-ray machine (Digital Diagnosis, Philips, Amsterdam, The Netherlands). The X-ray radiographs reflected the soft tissue swelling around the femoral head, osteolysis, deformity, and osteogenesis at necrosis sites.

\subsubsection{Histological Analysis}

After X-ray scanning, the rabbits were sacrificed at weeks 4 and 8 post-surgery, and the biopsies of implants and the adjacent tissues were obtained. Then the biopsies and tissues were fixed in formalin and embedded in paraffin for H\&E and Masson's Trichrome staining. The histological 
analysis combined with X-ray at the same necrosis site was used to investigate vascularization. The vessel density and mean vessel diameter were quantitatively analyzed by using ImageJ software.

\subsection{Statistical Analysis}

Unless indicated, data were given as mean $\pm \mathrm{SD}(n=5)$. Statistics analysis of obtained results was carried out using a one-way analysis of variance (one-way ANOVA), and significance reported at a $p$-value of $<0.05$ for $95 \%$ confidence. All statistical calculations were performed using the GraphPad Prism software.

\section{Results}

\subsection{Release Delivery of VEGF and MCP-1}

The VEGF and MCP-1 loaded CP-1 microspheres, VEGF loaded CP-1 microspheres, MCP-1 loaded CP-1 microspheres, VEGF loaded CP-2 microspheres, and MCP-1 loaded microspheres were prepared by a double emulsion method. SEM images showed that growth factors loaded microspheres were homogeneous and the diameters for the different kind of microspheres were $0.7 \pm 0.12 \mu \mathrm{m}$ (Figure S1). Then, the growth factor encapsulation efficiency $\left(E_{\text {encaps }}\right)$ was measured using ELISA kits, as shown in Figure S2. For dual VEGF and MCP-1 loaded into CP-1 or CP-2 microspheres, the $E_{\text {encaps }}$ of MCP-1 was higher than that of VEGF, and for a single growth factor loaded into CP-1 or CP-2 microspheres, the $E_{\text {encaps }}$ of MCP-1 was also higher than that of VEGF. According to the $E_{\text {encaps }}$ of VEGF and MCP-1, and the biodegradable characteristics of linear PLGA-mPEG block copolymer microspheres, three delivery systems were constructed by allocating different types of microspheres into star-shaped PLGA-mPEG hydrogels (Figure 1) [19]. Figure 1a showed that VEGF and MCP-1 had a similar release profile from the composite hydrogel in 4 weeks, proved that VEGF and MCP-1 were simultaneously released from the hydrogel. However, the cumulative MCP-1 release in delivery system II increased fast to $49.3 \pm 4.7 \%$ within the first 9 days compared to the delivery system I $(36.7 \pm 3.1 \%)$, exhibiting an initial burst release behavior (Figure 1b). For delivery system III, the cumulative MCP-1 release decreased to $16.8 \pm 3.9 \%$, presenting a delayed release behavior (Figure 1c). Meanwhile, VEGF performed a delay-release profile in delivery system II, which was similar to release profile of MCP-1 in delivery system III.

\subsection{In Vitro Cell Viability}

The VECs viability in composite hydrogels was quantitatively investigated by a CCK- 8 assay (Figure S3). The result showed that VECs viability increased over days in composite hydrogels with different delivery systems. Furthermore, VECs proliferated at a higher rate in the circumstance of delivery system II compared to others. The growth factor loaded microspheres dispersed in hydrogels uniformly which was confirmed by an optical microscope images analysis, and VECs expressed a formless morphology in composite hydrogels based on the fact that VECs could grow and reproduce in three-dimensional (3D) environments (Figure 2a-c). Fluorescence assay (DAPI/FITC) was also performed to evaluate the VECs proliferation in composite hydrogels, and the results were shown in Figure $2 \mathrm{~d}-\mathrm{f}$ where the cytoskeleton was represented by blue color and the nucleus was represented by blue color. Results displayed that the number of VECs in delivery system II and III was relatively higher than that of delivery system I. 


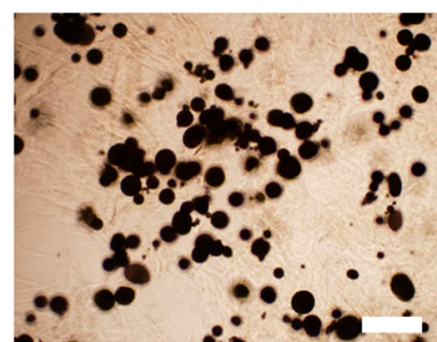

(a)

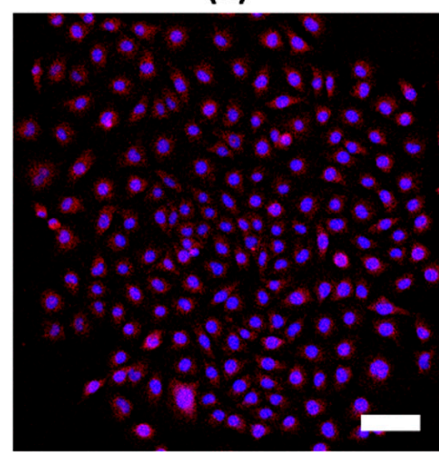

(d)

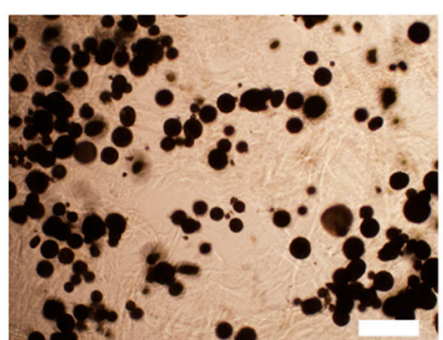

(b)

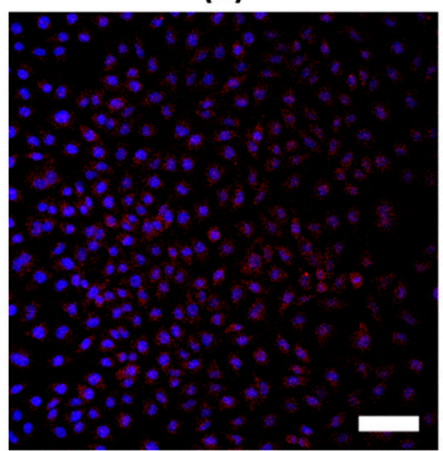

(e)

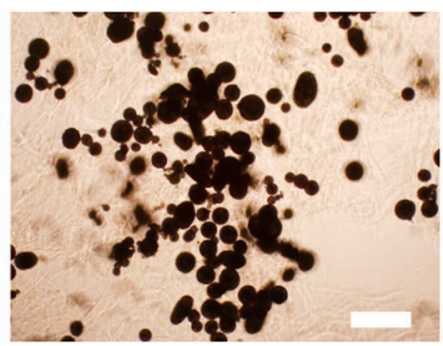

(c)

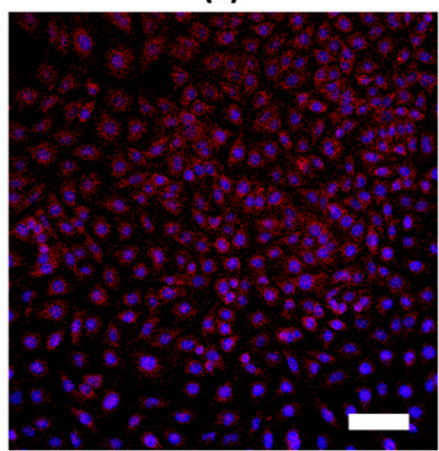

(f)

Figure 2. The VECs proliferation while cultured with composite hydrogels with different delivery systems at day 3. Optical microscope images of VECs in composite hydrogels with (a) delivery system I, (b) delivery system II, and (c) delivery system III. Meanwhile, the fluorescence images of VECs were obtained with (d) delivery system I, (e) delivery system II, and (f) delivery system III. The cell nucleus was represented by blue color, and cytoskeleton was represented by pink color. Scale bar: $25 \mu \mathrm{m}$.

\subsection{In Vivo Animal Study}

VECs-laden composite hydrogels with different delivery systems were implanted into necrosis site of femur head in Japanese rabbits by injection, all rabbits resumed normal ambulation and daily activity within one or two days after surgery. No signs of infection and wound split, and appetite loss was not observed for rabbits. Firstly, the host reaction around tissues and bone regeneration were evaluated by X-ray scanning and histological images, the results were presented in Figures 3 and 4. Figure 3a-d confirmed the successful construction of a femur head necrosis model by using the anhydrous alcohol method within 3 weeks. Soft tissue swelling around femur head was found before injection, and such swelling vanished after 4 weeks. The necrosis site couldn't be self-healed without intervention. However, new bone tissue was observed at the necrosis site with composite hydrogels treatment since week 4 , and new bone tissue was filled up at necrosis site completely at week 8 . The vessels density and mean vessel diameter were quantitatively obtained by ImageJ software according to H\&E and Masson staining results (20 images for each sample, which is the same necrosis site for the $X$-ray image), and the results were shown in Figure 5. The vessels density and mean vessel diameter in composite hydrogels with different delivery systems increased over the weeks compared to the control group. At week 4, the rabbits treated by composite hydrogel with delivery system II showed the highest value of the mean vessel diameter. However, at week 8 , the highest data of the vessel density and mean vessel diameter were observed in the delivery system III group. H\&E and Masson staining results confirmed that the new blood vessels were formed at the necrosis site of the rabbit femur head and that the ingrowth of new bone tissue was consistent with cell vessels density and mean vessel diameter. 


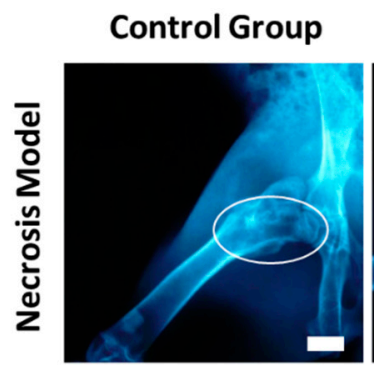

(a)

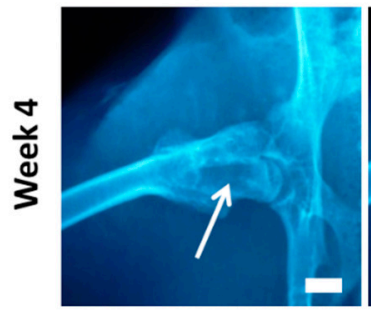

(e)

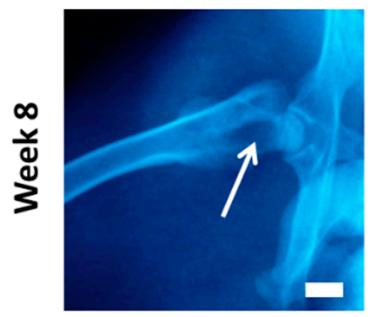

(i)
Delivery System I

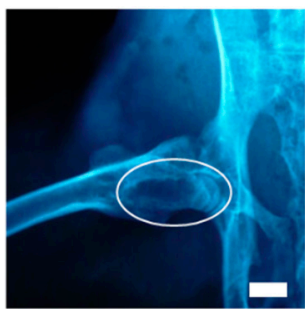

(b)

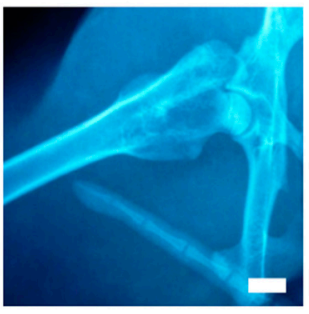

(f)

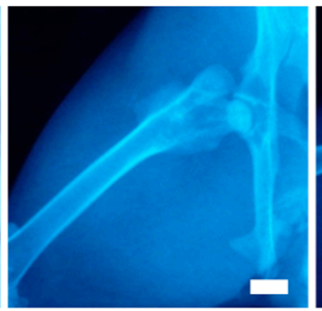

(j)

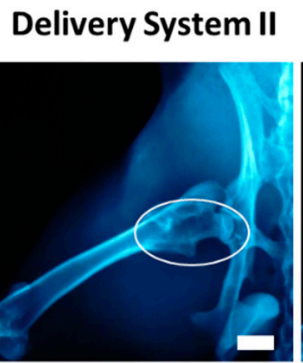

(c)

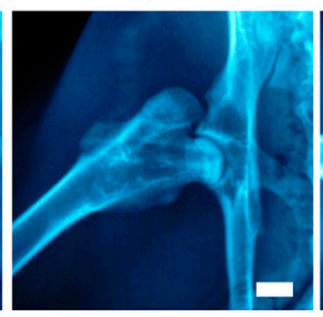

(g)

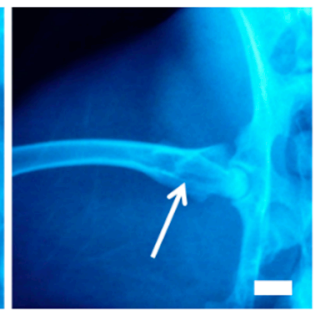

(k)
Delivery System III

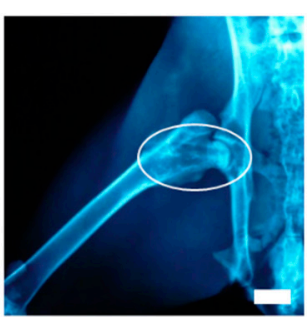

(d)

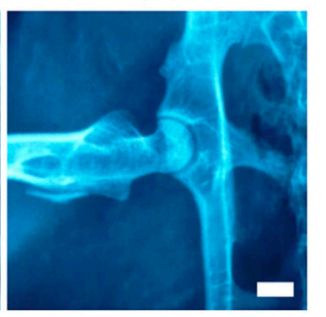

(h)

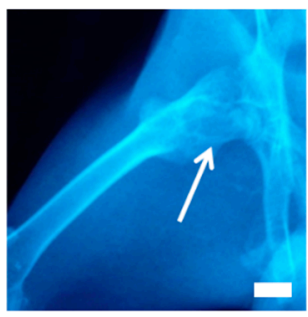

(I)

Figure 3. The X-ray images of the rabbit femur head in the right-posterior position at 4 th and 8th weeks post-operation. The femoral head necrosis models were constructed by using the bone injection of anhydrous alcohol method $(\mathbf{a}-\mathbf{d})$, the circles indicated the necrosis district. Non-intervention treatment as a control group, images (e,i) showed femur head without treatment (control group) at 4 th and 8th weeks respectively; images $(\mathbf{f}, \mathbf{j})$ showed femur head treated by composite hydrogel with delivery system I at 4th and 8th weeks respectively; images $(\mathbf{g}, \mathbf{k})$ showed femur head treated by composite hydrogel with delivery system II at 4 th and 8 th weeks respectively; images $(\mathbf{h}, \mathbf{l})$ showed femur head treated by composite hydrogel with delivery system III at 4th and 8th weeks, respectively. Scale bar: 1 $\mathrm{cm}$, the arrows indicated the regeneration district. 
H\&E Staining

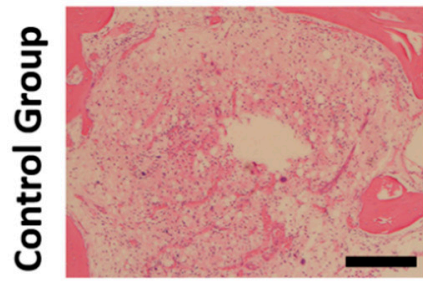

(a)

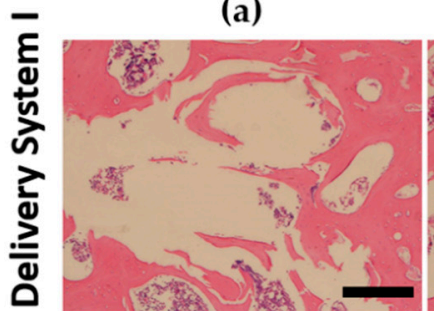

(e)

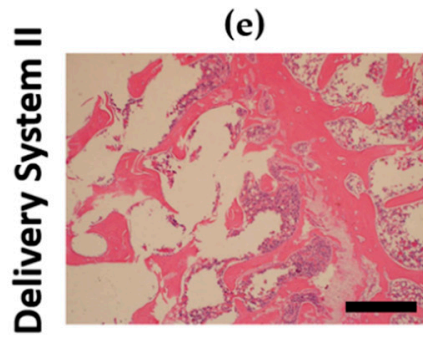

(i)

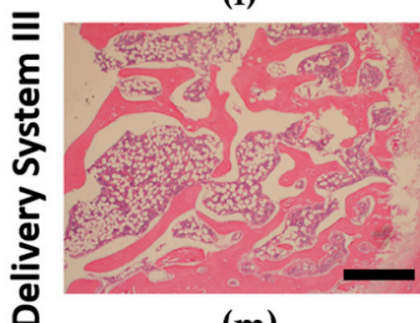

(m)

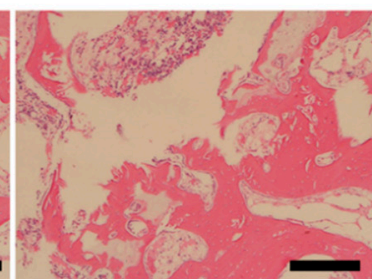

(b)

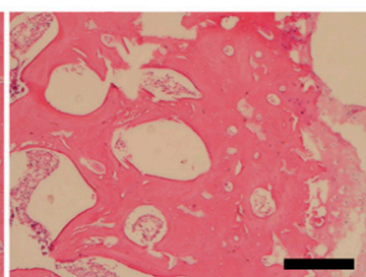

(f)

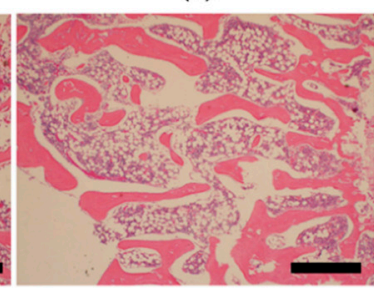

(j)

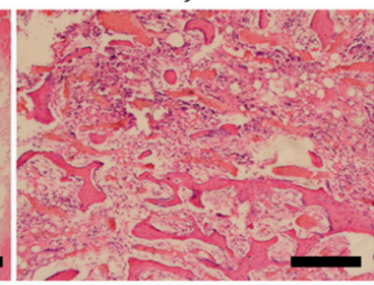

(n)
Masson Staining

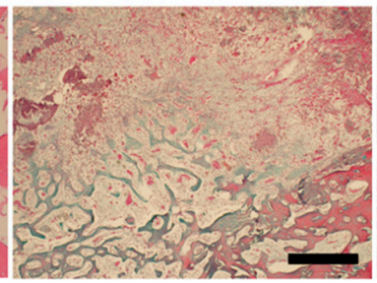

(c)

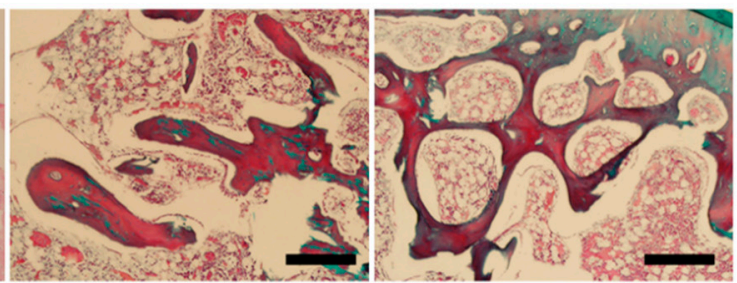

(g)

(h)

(d)
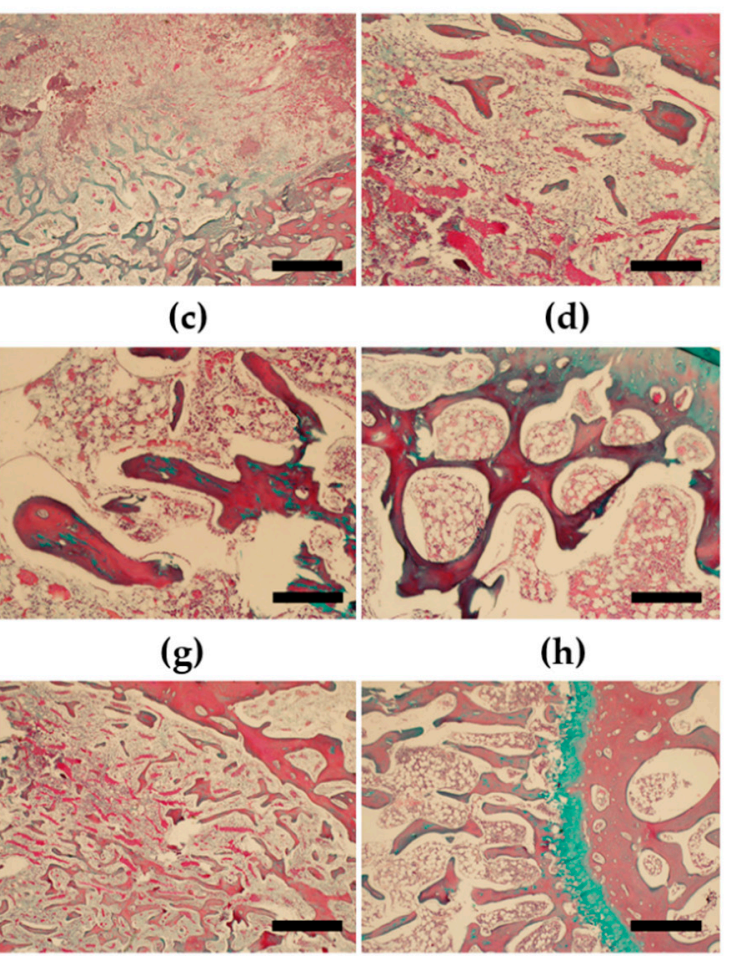

(k)

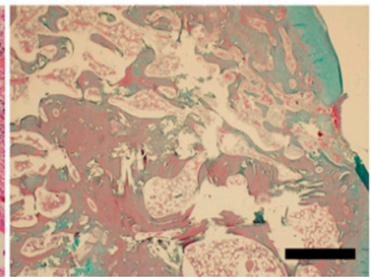

(o) (l)

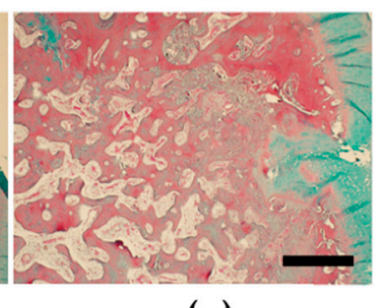

(p)

Figure 4. The representative optical images of H\&E and Masson staining slices of cross sections at the necrosis site where the composite hydrogels were implanted at the 4 th and 8 th weeks. $(\mathbf{a}, \mathbf{c})$ Control groups without intervention at 4 th weeks; $(\mathbf{b}, \mathbf{d})$ control groups without intervention at 8 th weeks; $(\mathbf{e}, \mathbf{g})$ the necrosis site treated by using composite hydrogels with delivery system I at 4 th week; $(\mathbf{f}, \mathbf{h})$ the necrosis site treated by using composite hydrogels with delivery system I at the 8 th week; $(\mathbf{i}, \mathbf{k})$ the necrosis site treated by using composite hydrogels with delivery system II at the 4th week; $(\mathbf{j}, 1)$ the necrosis site treated by using composite hydrogels with delivery system II at the 8 th week; $(\mathbf{m}, \mathbf{o})$ the necrosis site treated by using composite hydrogels with delivery system III at the 4th week; $(\mathbf{n}, \mathbf{p})$ the necrosis site treated by using composite hydrogels with delivery system III at the 8 th week. Scale bar: $100 \mu \mathrm{m}$. 


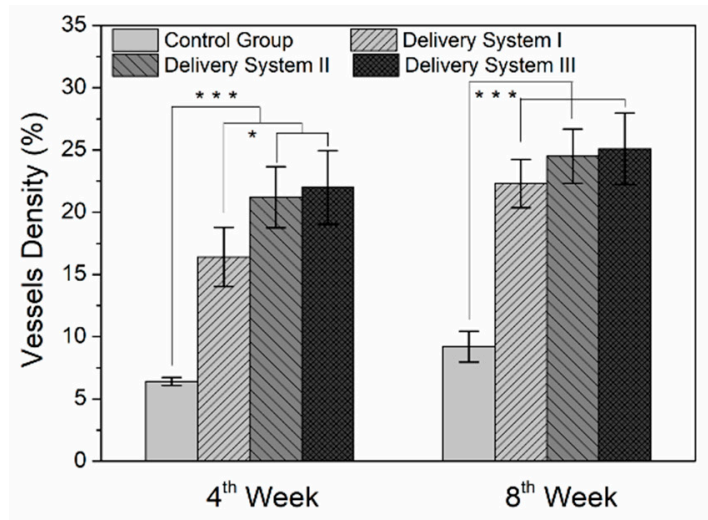

(a)

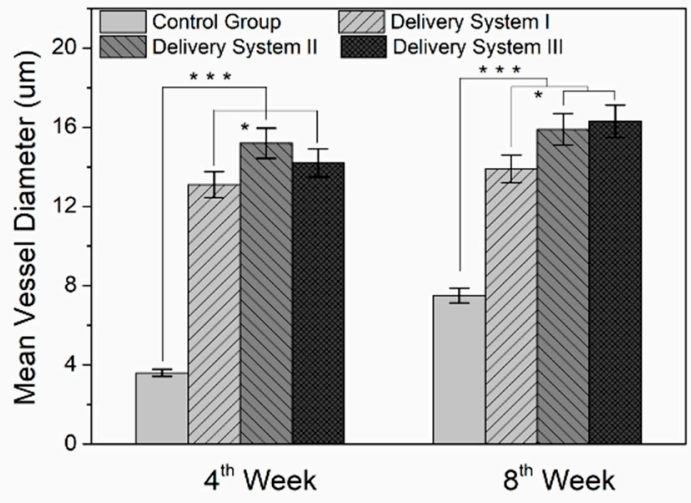

(b)

Figure 5. The vessels density and mean vessel diameter were analyzed by the ImageJ software quantitatively according to H\&E and Masson stained slices after the implantation of the composite hydrogels with different delivery systems into the necrosis site of femur head in rabbits at the 4th and 8th weeks. (a) Vessels density increased over weeks for three delivery systems; (b) mean vessel diameter also increased over weeks for three delivery systems, and delivery system III showed the highest value at the 4 th week. Non-intervention treatment as a control group $\left({ }^{* * *} p<0.001,{ }^{*} p<0.05\right)$.

\section{Discussion}

New vessels could be formed via the sprouting of existing vessels (angiogenesis) or the development of de novo vessels from progenitor cells (vasculogenesis), and during these complex processes, the suitable microenvironment which consists of cells, growth factors, and extracellular matrix (ECM) components could regulate both a nascent tube formation and vessel maturation [30,31]. The 3D scaffold encapsulated with cells and dual growth factors delivery system could mimic the natural microenvironment for vascularization and new bone formation in bone tissue engineering. Traditional growth factor administration caused high toxicity because of short half-lives of growth factor and slow penetration into tissue. Recently, the polymer microspheres have been widely used for growth factor storage and delivery efficiency. Our previous works showed that the linear poly(D,L-lactic-co-glycolic acid)-b-methoxy poly(ethylene glycol) (PLGA-mPEG) block copolymer microspheres had practical advantages on loading growth factor. For instance, the encapsulation efficiency could be regulated by the size of the microspheres, and release profile of growth factor could be adjusted by [LA]/[GA] ratio [19-21]. In this work, two linear PLGA-mPEG block copolymers with a different $[\mathrm{LA}] /[\mathrm{GA}]$ ratio (CP-1, CP-2) were used to prepare microspheres, the uniform growth factor loaded microspheres were obtained by the double emulsion method. The encapsulation efficiency of MCP-1 showed a higher value into CP-1 and CP-2 microspheres than that of VEGF (Figure S2). A higher initial loading with respect to the microspheres compared to the previous report (MCP-1, $1200 \mathrm{ng} / \mathrm{mg}$; VEGF, $1200 \mathrm{ng} / \mathrm{mg}$ ) was operated in this study, thus the release of both growth factors could remain for 4 weeks at least. While the growth factor loaded microspheres were composited into the hydrogel, the release of the growth factor was a little delayed from composite hydrogel compared to the bare microspheres [23,32]. However, the release profile of growth factor was mainly controlled by the degradability rate of the microspheres, thus different delivery systems could be facilely constructed by designing the proper formulation of microspheres co-loaded with VEGF and MCP-1, VEGF loaded microsphere, and MCP-1 loaded microsphere in hydrogels (Figure 1).

On the other hand, VECs transplantation was an effective approach for vascularization [4], and our previous works have proved that composite hydrogel encapsulated with VECs could enhance new blood vessel formation [23]. In this paper, both the linear PLGA-mPEG block copolymer for microsphere preparation and star-shaped PLGA-mPEG block copolymer for hydrogel preparation possessed a good cytocompatibility, thus, VECs survived in the composite hydrogel, and the viability of VECs also increased over days (Figure 2 and Figure S3). The growth factors alone could not promote 
the formation of the stable vasculature. So, it was a promising strategy to develop vascularization via the incorporation of VECs into composite hydrogel [33]. Cell-based approaches for therapeutic vascularization, including endothelial progenitor cell delivery, the engineered mesenchymal cell-based patches, and macrophage-based therapeutic strategies, have been studied for many years. However, many VECs-based approaches could not work effectively in clinical trials, which was not surprising as they lacked the proper delivery system of multiple growth factors due to the failure of recruiting and transplanting VECs [34,35]. Here, the synergistic effects of VECs and different simultaneous delivery systems of VEGF and MCP-1 on new vessel formation were primarily evaluated through in vivo animal experiments.

The star-shaped PLGA-mPEG block copolymer solution with an incorporation of the delivery system and VECs could be injected into a necrosis site of the femur head in Japanese rabbit. The composite hydrogel was formed in time with the help of animal body temperature. This technique was not only operated easily by researchers compared to traditional engineered scaffold replacement but also provided a new therapeutic strategy for treating chronic femoral head necrosis at an early stage instead of a total hip replacement $[23,27]$. Being benefited from the good histocompatibility of the composite (Figure 4), the composite hydrogel treatment did not affect the bodily functions of rabbits, and abnormal signs such as infection, host reaction were not detected. On the other hand, in the clinic, although magnetic resonance imaging showed many advantages at an early stage compared to X-rays, such as a high image quality, considering the lost-cost of the X-rays for patients, X-ray combined with a histological analysis were used in this study [36]. We previously explored the effect of the sustained release of VEGF on vessel formation [23]. In this study, we expanded single VEGF to the simultaneous release of VEGF and MCP-1. MCP-1 showed a distinct behavior in three delivery systems, thus, leading to observable differences at different delivery systems $\left({ }^{*} p<0.05\right)$, and significant differences compared to control group without intervention $(* * *<0.001)$. So, the release profile of MCP-1 played an important role in new vessel formation [7]. At week 8 , the vessel density and mean average diameter of vessels both increased. The data showed that the simultaneous release of VEGF and MCP-1 promoted mature vessels formation in comparison to a single VEGF release. Although new bones have emerged, some old bones and necrotic bones might still exist, above phenomena might cause the resurgence of diseases, and then increased second surgical risk. Thus, the sequential delivery of multiple growth factors still needs to be explored in the future. In addition, besides VECs, other types of cells such as stem cells and macrophages need to be loaded into composites with multiple growth factors delivery system for angiogenesis and osteogenesis for bone regeneration $[34,35,37]$.

\section{Conclusions}

In summary, the simultaneous delivery system for releasing VEGF and MCP-1 could be facilely constructed by using a linear PLGA-mPEG block copolymer microspheres carrier. The composited delivery system with VECs into star-shaped PLGA-mPEG block copolymer hydrogel; VECs showed a good proliferation in the composite hydrogel. Finally, the composite hydrogel was implanted into a necrosis site of rabbit femoral head by injection, the vessels density and mean vessels diameters increased over weeks which were confirmed by histological analysis. The present works advised that the simultaneous release of the VEGF and MCP-1 composite with VECs in hydrogels provided a potential strategy for therapeutic vascularization in bone tissue engineering. However, this strategy still bears further pre-clinical exploration in models of bone-related diseases.

Supplementary Materials: The following are available online at http:/ /www.mdpi.com/2076-3417/8/12/2438/ s1, Table S1: The designation of composite hydrogel with sequential release of VEGF and MCP-1, Figure S1: SEM images of VEGF and / or MCP-1 loaded CP-1 and CP-2 microspheres, Figure S2: The encapsulation efficiency of VEGF and/or MCP-1 in CP-1 and CP-2 microspheres, Figure S3: CCK-8 assay of VECs cultured with composite hydrogels for 1, 3 and 5 days.

Author Contributions: All authors discussed and agreed on the contents of the manuscript. Conceptualization, L.N. and P.C.; Methodology, L.N., H.Y., X.W. and P.C.; Formal Analysis, L.N., P.C., C.Z., Q.Z., P.G. and H.H.; Investigation, L.N., P.C., C.J., C.Z. and H.H.; Resources, L.N., P.C., C.J. and H.H.; Data Curation, L.N., P.C., C.J., 
X.W. and H.Y.; Writing-Original Draft Preparation, P.C., C.J. and X.W.; Writing-Review \& Editing, L.N., M.S., P.C. and X.W.

Funding: This research was funded by the National Natural Science Foundation of China No. 31700840, and the Key Scientific Research Project of Henan Province No. 18B430013.

Acknowledgments: This research was supported by the Nanhu Scholars Program for Young Scholars of XYNU. The authors would like to acknowledge Analysis \& Testing Center of XYNU for the use of their equipment. Finally, this work also thanks for Dong Chen from Department of Orthopedics at Wuhan Hanyang Hospital for animal model design, animal experiments and staining pictures analysis.

Conflicts of Interest: The authors declare no conflict of interest.

\section{References}

1. Rezaeeyazdi, M.; Colombani, T.; Memic, A.; Bencherif, S.A. Injectable hyaluronic Acid-co-Gelatin cryogels for tissue-engineering applications. Materials 2018, 11, 1374. [CrossRef] [PubMed]

2. Shrivats, A.R.; McDermott, M.C.; Hollinger, J.O. Bone tissue engineering: State of the union. Drug Discov. Today 2014, 19, 781-786. [CrossRef] [PubMed]

3. Gentile, P.; Ferreira, A.M.; Callaghan, J.T.; Miller, C.A.; Atkinson, J.; Freeman, C.; Hatton, P.V. Multilayer nanoscale encapsulation of biofunctional peptides to enhance bone tissue regeneration in vivo. Adv. Healthc. Mater. 2017, 6, 1601182. [CrossRef] [PubMed]

4. Kim, J.J.; EI-Fiqi, A.; Kim, H.W. Synergetic cues of bioactive nanoparticles and nanofibrous structure in bone scaffolds to stimulate osteogenesis and angiogenesis. ACS Appl. Mater. Interfaces 2017, 9, 2059-2073. [CrossRef] [PubMed]

5. $\quad$ Spiller, K.L.; Nassiri, S.; Witherel, C.E.; Anfang, R.R.; Ng, J.; Nakazawa, K.R.; Yu, T.; Vunjak-Novakovic, G. Sequential delivery of immunomodulatory cytokines to facilitate the M1-to-M2 transition of macrophages and enhance vascularization of bone scaffolds. Biomaterials 2015, 37, 194-207. [CrossRef] [PubMed]

6. Jay, S.M.; Shepherd, B.R.; Andrejecsk, J.W.; Kyriakides, T.R.; Pober, J.S.; Saltzman, W.M. Dual delivery of VEGF and MCP-1 to support endothelial cell transplantation for therapeutic vascularization. Biomaterials 2010, 31, 3054-3062. [CrossRef] [PubMed]

7. Cao, L.; Mooney, D.J. Spatiotemporal control over growth factor signaling for therapeutic neovascularization. Adv. Drug Deliver. Rev. 2007, 59, 1340-1350. [CrossRef] [PubMed]

8. Vo, T.N.; Kasper, F.K.; Mikos, A.G. Strategies for controlled delivery of growth factors and cells for bone regeneration. Adv. Drug Deliver. Rev. 2012, 64, 1292-1309. [CrossRef] [PubMed]

9. Simons, M.; Gordon, E.; Claesson-Welsh, L. Mechanisms and regulation of endothelial VEGF receptor signalling. Nat. Rev. Mol. Cell Biol. 2016, 17, 611. [CrossRef] [PubMed]

10. Kloepper, J.; Riedemann, L.; Amoozgar, Z.; Seano, G.; Susek, K.; Yu, V.; Dalvie, N.; Amelung, R.L.; Datta, M.; Song, J.W.; et al. Ang-2/VEGF bispecific antibody reprograms macrophages and resident microglia to anti-tumor phenotype and prolongs glioblastoma survival. Proc. Natl. Acad. Sci. USA 2016, 201525360. [CrossRef] [PubMed]

11. Lange, C.; Storkebaum, E.; de Almodóvar, C.R.; Dewerchin, M.; Carmeliet, P. Vascular endothelial growth factor: A neurovascular target in neurological diseases. Nat. Rev. Neurol. 2016, 12, 439. [CrossRef] [PubMed]

12. Dor, Y.; Djonov, V.; Abramovitch, R.; Itin, A.; Fishman, G.I.; Carmeliet, P.; Goelman, G.; Keshet, E. Conditional switching of VEGF provides new insights into adult neovascularization and pro-angiogenic therapy. EMBO J. 2002, 21, 1939-1947. [CrossRef] [PubMed]

13. Beamer, B.; Hettrich, C.; Lane, J. Vascular endothelial growth factors: An essential component of angiogenesis and fracture healing. HSS J. 2010, 6, 85-94. [CrossRef] [PubMed]

14. Klontzas, M.E.; Kenanidis, E.I.; MacFarlane, R.J.; Michail, T.; Potoupnis, M.E.; Heliotis, M.; Mantalaris, A.; Tsiridis, E. Investigational drugs for fracture healing: Preclinical \& clinical data. Expert Opin. Inv. Drug. 2016, 25, 585-596. [CrossRef]

15. Barati, D.; Shariati, S.R.P.; Moeinzadeh, S.; Melero-Martin, J.M.; Khademhosseini, A.; Jabbari, E. Spatiotemporal release of BMP-2 and VEGF enhances osteogenic and vasculogenic differentiation of human mesenchymal stem cells and endothelial colony-forming cells co-encapsulated in a patterned hydrogel. J. Control. Release 2016, 223, 126-136. [CrossRef] [PubMed] 
16. Richardson, T.P.; Peters, M.C.; Ennett, A.B.; Mooney, D.J. Polymeric system for dual growth factor delivery. Nat. Biotechnol. 2001, 19, 1029. [CrossRef] [PubMed]

17. Amirian, J.; Linh, N.T.B.; Min, Y.K.; Lee, B.T. Bone formation of a porous Gelatin-Pectin-biphasic calcium phosphate composite in presence of BMP-2 and VEGF. Int. J. Biol. Macromol. 2015, 76, 10-24. [CrossRef] [PubMed]

18. Awada, H.K.; Johnson, N.R.; Wang, Y. Sequential delivery of angiogenic growth factors improves revascularization and heart function after myocardical infarction. J. Control. Release 2015, 207, 7-17. [CrossRef] [PubMed]

19. Feng, S.; Nie, L.; Zou, P.; Suo, J. Effects of drug and polymer molecular weight on drug release from PLGA-mPEG microspheres. J. Appl. Polym. Sci. 2015, 132. [CrossRef]

20. Nie, L.; Zhang, G.; Hou, R.; Xu, H.; Li, Y.; Fu, J. Controllable promotion of chondrocyte adhesion and growth on PVA hydrogels by controlled release of TGF- $\beta 1$ from porous PLGA microspheres. Colloid Surface B 2015, 125, 51-57. [CrossRef] [PubMed]

21. Farokhi, M.; Mottaghitalab, F.; Shokrgozar, M.A.; Ou, K.L.; Mao, C.; Hosseinkhani, H. Importance of dual delivery systems for bone tissue engineering. J. Control. Release 2016, 225, 152-169. [CrossRef] [PubMed]

22. Kang, M.S.; Kim, J.H.; Singh, R.K.; Jang, J.H.; Kim, H.W. Therapeutic-designed electrospun bone scaffolds: Mesoporous bioactive nanocarriers in hollow fiber composites to sequentially deliver dual growth factors. Acta Biomater. 2015, 16, 103-116. [CrossRef] [PubMed]

23. Chen, D.; Zhang, C.; Huo, H.; Ji, C.; Sun, M.; Nie, L. Injectable temperature-sensitive hydrogel with VEGF loaded microspheres for vascularization and bone regeneration of femoral head necrosis. Mater. Lett. 2018, 229, 138-141. [CrossRef]

24. Nie, L.; Zou, P.; Feng, S.; Suo, J. Temperature-sensitive star-shaped block copolymers hydrogels for an injection application: Phase transition behavior and biocompatibility. J. Mater. Sci. Mater. Med. 2013, 24, 689-700. [CrossRef] [PubMed]

25. Nie, L.; Chen, D.; Suo, J.; Zou, P.; Feng, S.; Yang, Q.; Yang, S.; Ye, S. Physicochemical characterization and biocompatibility in vitro of biphasic calcium phosphate/polyvinyl alcohol scaffolds prepared by freeze-drying method for bone tissue engineering applications. Colloid Surface B 2012, 100, 169-176. [CrossRef] [PubMed]

26. Nie, L.; Chen, D.; Fu, J.; Yang, S.; Hou, R.; Suo, J. Macroporous biphasic calcium phosphate scaffolds reinforced by poly-L-lactic acid/hydroxyapatite nanocomposite coatings for bone regeneration. Biochem. Eng. J. 2015, 98, 29-37. [CrossRef]

27. Tozzi, G.; De Mori, A.; Oliveira, A.; Roldo, M. Composite hydrogels for bone regeneration. Materials 2016, 9 , 267. [CrossRef] [PubMed]

28. Mapara, M.; Thomas, B.S.; Bhat, K.M. Rabbit as an animal model for experimental research. Dent. Res. J. 2012, 9, 111c118. [CrossRef]

29. Shalumon, K.T.; Kuo, C.Y.; Wong, C.B.; Chien, Y.M.; Chen, H.A.; Chen, J.P. Gelatin/nanohydroxyapatite cryogel embedded poly(lactic-co-glycolic acid)/nanohydroxyapatite microsphere hybrid scaffolds for simultaneous bone regeneration and load-bearing. Polymers 2018, 10, 620. [CrossRef]

30. Carmeliet, P.; Jain, R.K. Molecular mechanisms and clinical applications of angiogenesis. Nature 2011, 473, 298-307. [CrossRef] [PubMed]

31. Moulisová, V.; Gonzalez-García, C.; Cantini, M.; Rodrigo-Navarro, A.; Weaver, J.; Costell, M.; Serra, R.S.; Dalby, M.J.; García, A.J.; Salmerón-Sánchez, M. Engineered microenvionments for synergistic VEGF-integrin signaling during vascularization. Biomaterials 2017, 126, 61-74. [CrossRef] [PubMed]

32. Qu, J.; Wang, L.; Niu, L.; Lin, J.; Huang, Q.; Jiang, X.; Li, M. Porous silk fibroin microspheres sustainably releasing bioactive basic fibroblast growth factor. Materials 2018, 11, 1280. [CrossRef] [PubMed]

33. Cao, R.; Bråkenhielm, E.; Pawliuk, R.; Wariaro, D.; Post, M.J.; Wahlberg, E.; Leboulch, P.; Cao, Y. Angiogenic synergism, vascular stability and improvement of hind-limb ischemia by a combination of PDGF-BB and FGF-2. Nat. Med. 2003, 9, 604-613. [CrossRef] [PubMed]

34. Boccardo, S.; Gaudiello, E.; Melly, L.; Cerino, G.; Ricci, D.; Martin, I.; Eckstein, F.; Banfi, A.; Marsano, A. Engineered mesenchymal cell-based patches as controlled VEGF delivery systems to induce extrinsic angiogenesis. Acta Biomater. 2016, 42, 127-135. [CrossRef] [PubMed]

35. Spiller, K.L.; Koh, T.J. Macrophage-based therapeutic strategies in regenerative medicine. Adv. Drug Deliver. Rev. 2017, 122, 74-83. [CrossRef] [PubMed] 
36. Baskin, E.; Dinur, T.; Lebel, E.; Tiomkin, M.; Elstein, D.; Zimran, A. Comparison of bone mineral density by dual-energy $\mathrm{X}$-ray absorptiometry and bone strength by speed-of-sound ultrasonography in adults with Gaucher disease. J. Clin. Densitom. 2016, 19, 465-470. [CrossRef] [PubMed]

37. Andrejecsk, J.W.; Chang, W.G.; Pober, J.S.; Saltzman, W.M. Controlled protein delivery in the generation of microvascular networks. Drug Deliv. Transl. Res. 2015, 5, 75-88. [CrossRef] [PubMed] 